\title{
Evidências de validade de uma versão brasileira da Fear of COVID-19 Scale
}

\author{
Evidence of the validity of a Brazilian version \\ of the Fear of COVID-19 Scale
}

Rodrigo Sanches Peres (https://orcid.org/0000-0002-2957-7554) ${ }^{1}$

Loriane Trombini Frick (https://orcid.org/0000-0002-4151-4329) ${ }^{2}$

Francine Nathalie Ferraresi Rodrigues Queluz (https://orcid.org/0000-0002-8869-6879) ${ }^{3}$

Sheyla Christine Santos Fernandes (https://orcid.org/0000-0003-4759-1314) ${ }^{4}$

Sidnei Rinaldo Priolo Filho (https://orcid.org/0000-0003-1320-9674) ${ }^{5}$

Ana Carina Stelko-Pereira (https://orcid.org/0000-0002-8089-132X) ${ }^{2}$

Jucimara Zacarias Martins (https://orcid.org/0000-0001-6800-3468) ${ }^{6}$

João Paulo Araújo Lessa (https://orcid.org/0000-0003-0751-7662) ${ }^{7}$

Heila Magali da Silva Veiga (https://orcid.org/0000-0002-7429-8124) ${ }^{1}$

Pedro Afonso Cortez (https://orcid.org/0000-0003-0107-2033) ${ }^{8}$

${ }^{1}$ Universidade Federal de Uberlândia. Av. Pará 1720, Bloco 2C, Umuarama. 38400-902

Uberlândia MG Brasil. rodrigosanchesperes@ufu.br

${ }^{2}$ Universidade Federal do

Paraná. Curitiba PR Brasil.

${ }^{3}$ Centro Universitário

Adventista de São Paulo.

Hortolândia SP Brasil.

${ }^{4}$ Universidade Federal de

Alagoas. Maceió AL Brasil.

${ }^{5}$ Universidade Tuiuti do

Paraná. Curitiba PR Brasil.

${ }^{6}$ Centro Universitário

Unigran Capital. Campo

Grande MS Brasil.

${ }^{7}$ Universidade Anhembi

Morumbi. São Paulo SP

Brasil.

${ }^{8}$ Universidade Metodista de

São Paulo. São Bernardo do

Campo SP Brasil.

\begin{abstract}
This study sought to analyze the evidence of the validity of a Brazilian version of the Fear of COVID-19 Scale (FCV-19S), based on indicators relating to: (1) the internal structure; (2) the internal consistency; (3) the relation with external variables; and (4) the content. The instrument was culturally adapted, focusing on semantic and specifically Brazilian linguistic aspects. The Brazilian version of the FCV-19S was then applied, in a virtual environment, to 211 participants $(72.98 \%$ female) with an average age of 37.07 years $(S D=13.03)$, together with the Self-Perception Questionnaire on Mental Health in Pandemics and the Sociodemographic and Functional Questionnaire. The confirmatory factor analysis revealed one-dimensionality. The internal consistency indices obtained (Cronbach's alpha $=0.921 ;$ McDonald's omega $=0.926$ ) can be considered high. The correlation between fear and obsessive thinking of the disease, generalized anxiety, generalized stress, phobic-avoidant behavior and bereavement due to the pandemic was found to be statistically significant. Evidence of the validity related to content, derived from a qualitative approach, were satisfactory. The conclusion drawn is that the Brazilian version of the $F C V$-19S proved to be adequate regarding the evidence of the expected validity.
\end{abstract}

Key words Fear, Validation Studies, COVID-19, Pandemics
Resumo O presente estudo teve como objetivo analisar evidências de validade de uma versão brasileira da Fear of COVID-19 Scale (FCV-19S), com base em indicadores concernentes: (1) à estrutura interna; (2) à consistência interna; (3) à relação com variáveis externas; e (4) ao conteúdo. Procedeu-se a adaptação cultural do instrumento, com foco em aspectos semânticos e linguísticos próprios do Brasil. A seguir, esta versão brasileira da FCV-19S foi aplicada, em um ambiente virtual, em 211 participantes $(72,98 \%$ do sexo feminino), com idade média de 37,07 anos ( $D P=13,03)$, juntamente com o Questionário de Autopercepção de Saúde Mental em Pandemia e o Questionário Sociodemográfico e Funcional. A análise fatorial confirmatória atestou unidimensionalidade. Os indices de consistência interna obtidos (alfa de Cronbach =0,921; ômega de McDonald =0,926) podem ser considerados elevados. Constatou-se correlação estatisticamente significativa entre medo e pensamento obsessivo, ansiedade generalizada, estresse generalizado, comportamento fóbico-evitativo e vivência de luto pela pandemia. As evidências de validade relativas ao conteúdo, oriundas de uma abordagem qualitativa, foram satisfatórias. Conclui-se que esta versão brasileira da FCV-19S mostrou-se adequada quanto às evidências de validade contempladas.

Palavras-chave Medo, Estudos de validação, COVID-19, Pandemias 


\section{Introdução}

O Brasil se tornou um epicentro emergente da pandemia de COVID-19 - doença potencialmente letal causada pelo novo coronavírus (SARS-CoV-2) - poucos meses após o registro dos primeiros casos no país ${ }^{1}$. Nesse cenário epidemiológico alarmante, diversas consequências psicológicas têm ocorrido, gerando como desdobramento importantes agravos à saúde mental ${ }^{2}$. A elevada taxa de transmissibilidade da doença e a existência de muitos entraves no que se refere à implementação de uma campanha de vacinação abrangente no território brasileiro ${ }^{3}$ são determinantes para tanto. Contudo, o impacto do distanciamento social e do isolamento social - medidas indicadas para conter a dispersão do referido patógeno - também deve ser considerado, pois pode deteriorar as relações interpessoais e a qualidade de vida, como alerta Roychowdhury ${ }^{4}$.

As consequências psicológicas da pandemia de COVID-19 tendem se revelar por meio de manifestações distintas daquelas que costumam ser observadas em contextos de saúde regulares e possivelmente se estenderão para além do período de disseminação descontrolada do SARS-CoV-25,6. Portanto, evidencia-se que o gerenciamento dos cuidados em saúde mental é essencial na atualidade, bem como demanda instrumentos de avaliação específicos ${ }^{7}$. É preciso ainda levar em conta que o medo - enquanto sensação proveniente da percepção de estímulos ameaçadores ${ }^{8}$ - se mostrou uma variável de grande relevância em crises sanitárias anteriores e ilustra como as consequências psicológicas da pandemia de COVID-19 podem se revestir de características próprias.

Afinal, muitas pessoas temem adoecer e morrer em função da doença, mas igualmente sentem medo quanto ao risco de infectar aqueles com quem convivem, quanto à eventual perda de seus meios de subsistência e quanto às possíveis restrições relacionais resultantes do distanciamento social ou do isolamento social, por exemplo ${ }^{10}$. Observam-se, adicionalmente, preocupações com as ameaças de colapso do sistema de saúde e de falta de alimentos, dentre outras, o que também suscita medo ${ }^{11}$. Nesse sentido, em consonância com Ornell et al. ${ }^{9}$, é possível afirmar que, paralelamente à pandemia de COVID-19, está em curso uma pandemia de medo.

O medo foi identificado como um importante preditor da adesão aos comportamentos preventivos preconizados pelas autoridades sanitá- rias para reduzir a transmissão do SARS-CoV-2 ${ }^{12}$. Já pessoas que apresentam baixa percepção individual de risco quanto à infecção pelo referido patógeno são propensas à imprudência ${ }^{13}$. E sabese que o medo desencadeado pela pandemia de COVID-19, por um lado, aumenta os níveis de ansiedade e estresse em indivíduos saudáveis e, por outro lado, piora o quadro clínico de pacientes com transtornos mentais pré-existentes ${ }^{14}$. Ou seja: o medo pode ser compreendido como uma emoção potencialmente adaptativa desencadeada por eventos que representam perigo, mas, quando excessivo ou insuficiente, tende a suscitar atitudes disfuncionais, nos planos individual e coletivo $^{11}$.

Portanto, um instrumento válido, direcionado à avaliação do medo relativo especificamente à COVID-19, seria capaz de proporcionar subsídios de grande utilidade para a prática dos profissionais de saúde como um todo. Com o intuito de preencher uma lacuna existente na literatura científica internacional à época, Ahorsu et al. ${ }^{15}$ desenvolveram a Fear of COVID-19 Scale (FCV -19S). Tal escala foi identificada por meio de uma revisão ${ }^{7}$ que resultou na composição de uma força-tarefa voltada à adaptação cultural e à validação, para o Brasil, de instrumentos para o rastreio de agravos à saúde mental em situações de crise sanitária. O presente estudo se afigura como uma das produções dessa força-tarefa.

Ressalte-se que a FCV-19S é um instrumento breve, de autorrelato, e sua versão original demonstrou possuir estrutura unidimensional estável e indicadores robustos quanto a consistência interna (alfa de Cronbach $=0,82$ ) e confiabilidade (Coeficiente de Correlação Intraclasse $=0,72)^{15}$. Deve-se sublinhar também que tal escala se encontra validada e adaptada em diversos idiomas $^{16-20}$. Sendo assim, o presente estudo teve como objetivo analisar evidências de validade de uma versão brasileira da FCV-19S, com base em indicadores concernentes: (1) à estrutura interna; (2) à consistência interna; (3) à relação com variáveis externas; e (4) ao conteúdo. Tendo em vista esse objetivo, faz-se necessário informar que, quando da finalização das atividades da força-tarefa citada anteriormente, uma primeira versão brasileira do referido instrumento foi proposta por outro grupo de pesquisadores ${ }^{21}$. Não obstante, no presente estudo, conforme será detalhado adiante, foram utilizados procedimentos metodológicos distintos, o que lhe confere originalidade. 
O presente estudo pode ser dividido em duas etapas, em função dos procedimentos metodológicos empregados. A Etapa 1 corresponde à adaptação cultural da FCV-19S, da qual foram extraídas evidências de validade relativas ao conteúdo, e foi constituída pela tradução, pela análise de contextualização e pela adequação semântica do instrumento. A Etapa 2 proporcionou indicadores referentes à estrutura interna e à consistência interna desta versão brasileira da FCV-19S e à relação do medo com variáveis externas, e será descrita aqui considerando-se seus participantes, instrumentos e estratégias de coleta e análise de dados.

\section{Etapa 1}

\section{Tradução}

A adaptação cultural da FCV-19S do inglês para o português brasileiro foi realizada com base nos procedimentos metodológicos descritos por Van de Vijver ${ }^{22}$ e teve como foco aspectos semânticos e linguísticos próprios do Brasil. Sendo assim, três tradutores (um tradutor profissional e dois profissionais de saúde bilíngues com conhecimento teórico sobre o tema) foram contatados via e-mail e solicitados a produzir três traduções independentes do instrumento. $\mathrm{Na}$ sequência, um comitê de especialistas composto por três professores universitários (todos doutores) da área da saúde realizou, qualitativamente, a análise de conteúdo dos itens e das opções de resposta das três traduções. Tal análise, em um primeiro momento, foi conduzida independentemente. Em um segundo momento, os integrantes do comitê de especialistas se reuniram para apresentar suas opiniões, o que foi feito por meio de um aplicativo de videoconferência. Indicações de ajustes foram introduzidas depois de discutidas em prol da obtenção de consenso, e assim chegou-se à versão pré-teste do instrumento.

\section{Análise de contextualização}

Para analisar a contextualização dos itens e das opções de resposta da versão pré-teste do instrumento em relação à população-alvo, recorreuse à realização de entrevistas cognitivas. Trata-se de uma valiosa ferramenta para investigar se um instrumento em adaptação cultural é interpretado ou não como esperado ${ }^{23}$. Mais precisamente, foi conduzida - também por meio de um aplicativo de videoconferência - uma entrevista cognitiva individual com cada um dos três respondentes (duas mulheres e um homem) intencionalmente selecionados por apresentarem diferentes faixas etárias (entre 30 e 50 anos de idade) e níveis de escolaridade (do ensino fundamental completo ao ensino superior completo). As entrevistas cognitivas, duraram, em média, 30 minutos.

Os respondentes foram localizados a partir de indicações provenientes dos círculos sociais dos pesquisadores e foram solicitados, durante as entrevistas cognitivas, a externar seus entendimentos sobre os itens e as opções de resposta da versão pré-teste do instrumento, bem como a indicar termos de difícil compreensão e sugerir possíveis alternativas, quando pertinente. $O$ pesquisador responsável registrou detalhadamente, por escrito, as opiniões dos respondentes, sendo que optou por não proceder gravações para que eles pudessem se sentir à vontade. Os dados oriundos das entrevistas cognitivas foram, então, apresentados pelo pesquisador responsável ao comitê de especialistas, novamente por meio de um aplicativo de videoconferência, no início da reunião em que foi promovida a adequação semântica.

\section{Adequação semântica}

A adequação semântica realizada pelo comitê de especialistas buscou aprimorar a compreensibilidade dos itens e das opções de resposta mediante a eventual introdução, nesta versão brasileira instrumento, de modificações consensualmente indicadas por seus integrantes após a análise qualitativa dos dados oriundos das entrevistas cognitivas. Cabe reforçar que, no presente estudo, a adaptação cultural da FCV-19S privilegiou aspectos semânticos e linguísticos próprios do Brasil, como mencionado, e não a plena equivalência em relação à sua versão original, redigida em inglês. Justamente por esse motivo, não foi empreendida a retrotradução do instrumento, expediente que, embora bastante difundido, pode levar à indesejada retenção de formas gramaticais típicas do idioma de origem ${ }^{24}$. Optouse, portanto, por uma abordagem funcionalista, a fim de garantir a coerência dos termos utilizados em face da realidade da população-alvo.

\section{Etapa 2}

\section{Participantes}

Participaram da Etapa 2 do presente estudo 211 brasileiros que se declararam economicamente ativos, isto é, que exerciam ou se encontravam em condições de exercer atividades profissionais. Em sua maioria, os participantes eram do sexo feminino $(72,98 \%)$, se enquadraram 
como latino-americanos brancos $(74,88 \%)$ e referiram que haviam cursado até o nível superior $(55,50 \%)$. Ressalte-se ainda que a idade média dos participantes foi de 37,07 anos ( $\mathrm{DP}=13,03)$. E deve-se esclarecer que o recrutamento dos participantes foi realizado de maneira online, por meio de um hyperlink divulgado nas redes sociais e institucionais dos integrantes da força-tarefa.

\section{Instrumentos}

$\mathrm{Na}$ Etapa 2 do presente estudo foram aplicados três instrumentos. O primeiro deles é a FCV -19S, já apresentada em termos de sua finalidade. Vale acrescentar que tal instrumento é composto por sete itens e oferece cinco opções de resposta, sendo que o escore total, calculado mediante a soma da pontuação de cada item, varia de sete a trinta e cinco. Ademais, é importante informar que a versão original da FCV-19S foi elaborada com base em uma extensa revisão que levou à identificação de diversos instrumentos voltados à avaliação do medo referente a doenças descritas anteriormente. Ahorsu et al..$^{15}$ extraíram desses instrumentos vinte e oito itens considerados particularmente relevantes e, a seguir, onze deles foram descartados por um comitê de especialistas. Os dezessete itens restantes foram avaliados por outro comitê de especialistas, o qual descartou mais sete itens, devido à similaridade de conteúdo. Obteve-se, assim, uma versão preliminar do instrumento, com dez itens. Essa versão foi utilizada em um estudo-piloto. Os três itens que apresentaram baixas correlações item-total foram retirados, de modo que a versão final do instrumento foi constituída por sete itens.

A fim de viabilizar o acesso a dados para a verificação de associações do medo com variáveis externas, na Etapa 2 do presente estudo também foi utilizado o Questionário de Autopercepção de Saúde Mental em Pandemia (QASMP), criado pelos integrantes da força-tarefa responsável por esta versão brasileira da FCV-19S especialmente para esta oportunidade. O QASMP se afigura como um instrumento breve, de autorrelato, e é composto por quatro itens que, com base na literatura especializada ${ }^{25}$, contemplam sinais e sintomas das seguintes condições: (1) comportamento fóbico-evitativo; (2) estresse generalizado; (3) ansiedade generalizada; e (4) pensamento obsessivo. É preciso esclarecer que tal instrumento oferece duas opções de resposta, de modo a identificar a presença ou a ausência dos sinais e sintomas em questão, e que, quanto à sua consistência interna, os valores obtidos foram os seguintes: alfa de Cronbach $=0,747$ e ômega de McDonald $=0,776$.
Por fim, igualmente foi empregado na Etapa 2 do presente estudo o Questionário Sociodemográfico e Funcional (QSF), outra criação dos integrantes da referida força-tarefa. A finalidade do referido instrumento foi subsidiar a caracterização dos participantes no tocante a: (1) idade; (2) gênero; (3) etnia; (4) escolaridade; (5) tempo de atuação no trabalho atual; (6) natureza profissional do cargo exercido; (7) redução salarial na pandemia; (8) adesão ao distanciamento social preconizado pelas autoridades sanitárias; (9) diagnóstico de COVID-19; (10) diagnóstico de transtorno mental; (11) convivência com pessoas infectadas pelo SARS-CoV-2 e (12) vivência de luto devido à pandemia. Para alguns itens, as respostas eram livres, ao passo que para outros, havia um número de opções de resposta determinado pela especificidade da informação que se buscava obter.

\section{Coleta de dados}

A coleta de dados foi realizada em um ambiente virtual, sendo que os participantes, após a visualização do Termo de Consentimento Livre e Esclarecido e a devida formalização de anuência, responderam aos instrumentos por meio de um aplicativo de formulários online. Vale destacar que tal estratégia de coleta de dados foi aprovada por um Comitê de Ética em Pesquisa e possibilitou o atendimento dos princípios éticos estabelecidos na legislação vigente para pesquisas com seres humanos no Brasil.

\section{Análise de dados}

Os dados coletados foram analisados com o emprego do software JASP $0.14 .0^{26}$. Estatísticas descritivas (média e desvio-padrão quanto à idade e tempo de atuação no trabalho atual $\mathrm{e}$ porcentagem quanto às demais características) foram adotadas para delinear o perfil dos participantes em termos sociodemográficos e funcionais. O índice Kaiser-Meyer-Olklin (KMO) e o teste de esfericidade de Bartlett foram inspecionados para aplicação da análise fatorial na matriz de dados ${ }^{27}$. A análise fatorial foi utilizada por meio de modelagem restrita com simulação do algoritmo Diagonally Weighted Least Squares (DWSL) do Mplus no JASP ${ }^{28}$, sendo que a carga fatorial mínima para retenção foi de 0,30 , como recomendam Hair et al. ${ }^{27}$.

As medidas de consistência interna selecionadas foram alfa de Cronbach e ômega de McDonald $^{29}$. A correlação de Pearson foi empregada para verificar o nível de associação de medo com variáveis externas, com o intuito de averiguar as 


\section{Resultados}

\section{Etapa 1}

No tocante à adaptação cultural da FCV-19S, ressalte-se que, de modo geral, as três traduções produzidas pelos tradutores foram consideradas satisfatórias, de acordo com a análise qualitativa executada pelo comitê de especialistas. Contudo, foi selecionada como base a tradução produzida pelo tradutor profissional, na qual os itens foram redigidos de forma padronizada quanto ao posicionamento de sujeito e predicado, o que lhes conferiu maior clareza. Nessa tradução somente duas expressões foram substituídas ("perder a minha vida" foi substituída por "morrer" e "úmidas" foi substituída por "suadas") pelo comitê de especialistas, pois as alternativas presentes nas outras duas traduções foram consensualmente consideradas mais diretas e, logo, potencialmente mais compreensíveis por parte da população-alvo. Sendo assim, a formulação da versão pré-teste do instrumento não demandou alterações de grande porte acerca do conteúdo.

Já durante as entrevistas cognitivas, dois dos três respondentes (aqueles com ensino fundamental completo e ensino médio completo, especificamente) apresentaram dificuldades para diferenciar as opções de resposta, então alusivas à concordância (de "discordo completamente" a "concordo completamente"), acompanhando aquelas adotadas na versão original da FCV-19S. Além disso, os três respondentes demonstraram não saber se o termo "coronavírus-19" - também empregado originalmente no instrumento - se referia à COVID-19 ou ao patógeno que a causa. Os respondentes, inclusive, sugeriram sua substituição pelo termo "pandemia", alegando que o primeiro, em contraste com o segundo, era pouco utilizado no cotidiano por eles mesmos e por aqueles que integram seus círculos sociais.

Levando em conta os dados oriundos das entrevistas cognitivas, o comitê de especialistas, durante a adequação semântica, procedeu dois

ajustes principais, e assim foi obtida esta versão brasileira da FCV-19S (Quadro 1). Um dos ajustes consistiu na inserção de opções de resposta alusivas à frequência (de "nunca" a "sempre") no lugar daquelas alusivas à concordância. E, em prol da compreensibilidade dos itens, o comitê de especialistas, por meio do outro ajuste, decidiu acatar a sugestão dos respondentes quanto à substituição do termo "coronavírus-19" por "pandemia". Cumpre assinalar que também respaldou a introdução desse ajuste o julgamento de que, com ele, as possibilidades de utilização do instrumento seriam ampliadas, pois o tornaria aproveitável tanto na pandemia de COVID-19 quanto, mediante eventuais readequações, em pandemias futuras.

\section{Etapa 2}

Os resultados obtidos por meio do QSF, para além daqueles já antecipados no tópico relativo aos participantes (idade, gênero, etnia e escolaridade), revelam que o tempo médio de atuação dos participantes no trabalho atual foi de 8,31 anos $(\mathrm{DP}=10,03)$ e que eles, predominantemente, exerciam cargos técnicos na própria área de formação $(30,69 \%)$, ou atuavam de modo autônomo e informal $(26,51 \%)$. Cerca de um quarto dos participantes $(24,88 \%)$ reportou ter sofrido redução salarial em decorrência da pandemia. A maioria declarou ter aderido ao isolamento social preconizado pelas autoridades sanitárias $(61,61 \%)$ e não ter recebido diagnóstico de $\mathrm{CO}$ VID-19 $(60,74 \%)$ ou de qualquer transtorno mental (80,57\%), sendo que $39,81 \%$ afirmaram conviver diretamente com pessoas infectadas pelo SARS-CoV-2 e 30,23\% mencionaram a vivência de luto em virtude da pandemia. Já em relação à autopercepção sobre a própria saúde mental no contexto pandêmico atual, avaliada por meio do QASMP, os participantes sinalizaram, em diferentes porcentagens, a ocorrência de comportamento fóbico-evitativo $(66,02 \%)$, estresse generalizado $(38,75 \%)$, ansiedade generalizada $(36,84 \%)$ e pensamento obsessivo $(36,36 \%)$.

A análise fatorial confirmatória subsidiou a obtenção dos seguintes índices de ajuste: $\chi 2=12,86 ; \mathrm{gl}=14 ; \mathrm{p}<0,53 ; \mathrm{TLI}=1,00 ; \mathrm{CFI}=1,00$; $\mathrm{SRMR}=0,06$. A adequação dos índices evidencia a unidimensionalidade desta versão brasileira da FCV-19S. A maior carga fatorial foi verificada no item $1(0,872)$, ao passo que a menor ocorreu no item $3(0,656)$, como se vê na Tabela 1 . Os valores obtidos quanto à consistência interna de tal ins- 
Quadro 1. Fear of COVID-19 Scale (Versão Brasileira).

\begin{tabular}{|c|c|c|c|c|c|}
\hline \multicolumn{6}{|c|}{$\begin{array}{l}\text { O presente instrumento avalia o medo de ficar doente por causa da pandemia. Para } \\
\text { responder, assinale uma das opções utilizando a escala de respostas apresentada a seguir. }\end{array}$} \\
\hline \multirow[t]{2}{*}{ Itens } & Nunca & $\begin{array}{c}\text { Poucas } \\
\text { vezes }\end{array}$ & $\begin{array}{l}\text { Algumas } \\
\text { vezes }\end{array}$ & $\begin{array}{c}\text { Muitas } \\
\text { vezes }\end{array}$ & Sempre \\
\hline & 1 & 2 & 3 & 4 & 5 \\
\hline $\begin{array}{l}\text { 1. Eu tenho muito medo de ficar doente por } \\
\text { causa da pandemia. }\end{array}$ & [ ] & [ ] & [ ] & [ ] & [ ] \\
\hline $\begin{array}{l}\text { 2. Eu fico incomodado(a) quando penso que } \\
\text { posso ficar doente por causa da pandemia. }\end{array}$ & [ ] & [ ] & [ ] & [ ] & [ ] \\
\hline $\begin{array}{l}\text { 3. Minhas mãos ficam suadas quando } \\
\text { eu penso no adoecimento causado pela } \\
\text { pandemia. }\end{array}$ & [ ] & [ ] & [ ] & [ ] & [ ] \\
\hline $\begin{array}{l}\text { 4. Eu tenho medo de morrer por causa da } \\
\text { pandemia. }\end{array}$ & [ ] & [ ] & [ ] & [ ] & [ ] \\
\hline $\begin{array}{l}\text { 5. Eu fico nervoso(a) ou ansioso(a) quando } \\
\text { vejo notícias ou histórias nas redes sociais } \\
\text { sobre o adoecimento causado pela pandemia. }\end{array}$ & [ ] & [ ] & [ ] & [ ] & [ ] \\
\hline $\begin{array}{l}\text { 6. Eu não consigo dormir porque estou } \\
\text { preocupado(a) em ficar doente por causa da } \\
\text { pandemia. }\end{array}$ & [ ] & [ ] & [ ] & [ ] & [ ] \\
\hline $\begin{array}{l}\text { 7. Meu coração acelera ou palpita quando eu } \\
\text { penso que posso ficar doente por causa da } \\
\text { pandemia. }\end{array}$ & [ ] & [ ] & {$[$ ] } & {$[$ ] } & [ ] \\
\hline
\end{tabular}

Fonte: Elaborado pelos autores.

Tabela 1. Estrutura fatorial e índices de consistência interna da FCV-19S (versão brasileira).

\begin{tabular}{lc}
\hline \multicolumn{1}{c}{ Itens } & $\begin{array}{c}\text { Cargas fatoriais } \\
\text { padronizadas }\end{array}$ \\
\hline 1 & 0,872 \\
2 & 0,853 \\
3 & 0,656 \\
4 & 0,815 \\
5 & 0,853 \\
6 & 0,689 \\
7 & 0,761 \\
alfa de Cronbach & 0,921 \\
ômega de McDonald & 0,926 \\
\hline
\end{tabular}

Fonte: Elaborado pelos autores.

trumento foram os seguintes: alfa de Cronbach $=0,921$ e ômega de McDonald $=0,926$. E, como se vê na Tabela 2, constatou-se correlação estatisticamente significativa entre medo relativo à pandemia vigente e todas as variáveis contempladas pelo QASMP. Ademais, optou-se por avaliar a correlação com a vivência de luto devido a COVID-19 conforme investigada a partir do
QSL, sendo que, também no que se refere a essa variável, foi constatada significância estatística. Portanto, escores mais elevados na FCV-19S demonstraram associação positiva com pensamento obsessivo $(\mathrm{r}=0,667)$, ansiedade generalizada $(r=0,572)$, estresse generalizado $(r=0,531)$, comportamento fóbico-evitativo $(\mathrm{r}=0,412)$ e vivência de luto pela pandemia $(\mathrm{r}=0,170)$.

\section{Discussão}

No que diz respeito a instrumentos em adaptação cultural, evidências de validade relativas ao conteúdo podem ser obtidas de diferentes formas, inclusive por meio de uma abordagem qualitativa ${ }^{31}$, como foi feito no presente estudo a fim de responder a um de seus objetivos. Tal abordagem, é pertinente recapitular, foi empregada pelo comitê de especialistas tanto para avaliar as traduções produzidas pelos tradutores quanto para analisar os dados oriundos das entrevistas cognitivas. Na pesquisa que gerou uma primeira versão brasileira da FCV-19S ${ }^{21}$, em contrapartida, foi privilegiada uma abordagem quantitativa a 
Tabela 2. Correlações entre medo relativo à pandemia e variáveis externas.

\begin{tabular}{|c|c|c|c|c|c|c|}
\hline Variáveis & Medo & $\begin{array}{l}\text { Comportamento } \\
\text { fóbico-evitativo }\end{array}$ & $\begin{array}{c}\text { Estresse } \\
\text { generalizado }\end{array}$ & $\begin{array}{c}\text { Ansiedade } \\
\text { generalizada }\end{array}$ & $\begin{array}{c}\text { Pensamento } \\
\text { obsessivo }\end{array}$ & $\begin{array}{c}\text { Vivência } \\
\text { de luto } \\
\text { pela } \\
\text { pandemia }\end{array}$ \\
\hline Medo & - & & & & & \\
\hline $\begin{array}{l}\text { Comportamento } \\
\text { fóbico-evitativo }\end{array}$ & $0,412^{* * *}$ & - & & & & \\
\hline Estresse generalizado & $0,531^{\star * *}$ & $0,218^{\star *}$ & - & & & \\
\hline Ansiedade generalizada & $0,572^{\star * *}$ & $0,192^{\star *}$ & $0,757^{\star * *}$ & - & & \\
\hline Pensamento obsessivo & $0,667^{\star * *}$ & $0,332^{\star * *}$ & $0,542^{\star * *}$ & $0,495^{\star * *}$ & - & \\
\hline $\begin{array}{l}\text { Vivência de luto pela } \\
\text { pandemia }\end{array}$ & $0,170^{*}$ & 0,067 & 0,102 & 0,065 & 0,094 & - \\
\hline
\end{tabular}

${ }^{*} \mathrm{p}<.05,{ }^{* *} \mathrm{p}<.01,{ }^{* * *} \mathrm{p}<.001$.

Fonte: Elaborado pelos autores.

propósito da obtenção de evidências de validade relativas ao conteúdo, e foi procedida a retrotradução do instrumento. Mas cabe reforçar que a indesejada retenção de formas gramaticais típicas do idioma de origem se afigura como um dos possíveis efeitos da retrotradução ${ }^{24}$, razão pela qual, no presente estudo, esse expediente não foi adotado.

Ademais, a pesquisa que gerou uma primeira versão brasileira da $\mathrm{FCV}-19 \mathrm{~S}^{21}$ prescindiu da realização de entrevistas cognitivas, sendo que a compreensibilidade dos itens foi avaliada apenas por meio da inclusão de uma opção de resposta adicional ("não entendi") na versão pré-teste do instrumento. E foi a partir dos dados obtidos com essa estratégia que, na referida pesquisa, os pesquisadores concluíram que houve compreensão por parte dos respondentes em relação ao termo "COVID-19", utilizado nos itens ao invés do termo "coronavírus-19”, empregado na versão original da FCV-19S. Já no presente estudo, como mencionado, a adequação semântica realizada pelo comitê de especialistas incorporou as sugestões dos respondentes veiculadas nas entrevistas cognitivas, de modo que o termo "coronavírus-19" foi substituído por "pandemia”. Como consequência, esta versão brasileira da FCV-19S pode favorecer respostas rápidas no que diz respeito à disponibilização de instrumentos para o rastreio de agravos à saúde mental em novas situações de crise sanitária, na linha do que preconizam Hubley e Zumbo ${ }^{32}$ e Ågerfalk et al. ${ }^{33}$.

Portanto, parece razoável propor que os procedimentos metodológicos utilizados no presente estudo quanto à adaptação cultural asseguram a validade de conteúdo desta versão brasileira da FCV-19S e, adicionalmente, lhe conferem um diferencial quanto às perspectivas de utilização em contextos pandêmicos futuros. As evidências de validade relativas à estrutura interna também podem ser consideradas satisfatórias, pois, conforme exposto anteriormente, a análise fatorial confirmatória atestou que esta versão brasileira da FCV-19S, a exemplo de sua versão original ${ }^{15}$, é unidimensional. Tal achado corresponde àqueles provenientes de pesquisas sobre o instrumento em outros idiomas, dentre os quais o árabe ${ }^{16}$, o espanhol $^{17}$, o italiano ${ }^{18}$, o japonês ${ }^{19}$ e o malaio ${ }^{20}$. A pesquisa que gerou uma primeira versão brasileira da FCV-19S ${ }^{21}$, todavia, indicou, inesperadamente, a existência de dois fatores.

Deve-se recordar que, quanto à consistência interna, os valores obtidos no presente estudo foram os seguintes: alfa de Cronbach $=0,921 \mathrm{e}$ ômega de McDonald =0,926. Em pesquisas internacionais voltadas à validação de versões da FCV-19S ${ }^{16-20}$, tais indicadores, respectivamente, variaram de 0,835 a 0,893 e de 0,894 a 0,940 . Portanto, os valores ora reportados são compatíveis com aqueles previamente divulgados por outros pesquisadores, além de que podem ser considerados elevados e, assim, constituem evidências de consistência interna desejável ${ }^{34}$. Na pesquisa que gerou uma primeira versão brasileira da FC$\mathrm{V}-19 S^{21}$, apenas uma medida de tal propriedade psicométrica foi calculada, produzindo, não obstante, um valor também classificável como elevado (alfa de Cronbach $=0,859$ ).

É preciso frisar que, no presente estudo, as evidências de validade concernentes à relação do medo com variáveis externas igualmente se mostraram adequadas, pois havia a expectativa de associação positiva com sinais e sintomas de agravos à saúde mental e, adicionalmente, com 
a vivência de luto devido a COVID-19, o que de fato foi verificado. Em linhas gerais, pesquisas prévias fornecem respaldo para tais associações. Na pesquisa por meio da qual a FCV-19S foi originalmente apresentada ${ }^{15}$, apurou-se correlação entre medo e ansiedade ( $\mathrm{r}=0,511)$, condição avaliada, no caso, por meio da Hospital Anxiety and Depression Scale (HADS). Cumpre assinalar que, por desconsiderar sinais e sintomas de natureza somática, a HADS vem sendo amplamente empregada junto a pacientes com doenças orgânicas, provenientes de diferentes serviços de saúde, e não apenas de hospitais, em contraste com o que sugere seu nome ${ }^{35}$.

Conforme informado previamente, o medo desencadeado pela pandemia de COVID-19 tende a piorar o quadro clínico de pacientes com transtornos mentais pré-existentes ${ }^{14}$. Tal fenômeno pode ser observado com clareza no transtorno obsessivo-compulsivo, já que um de seus sintomas é a repetição exagerada de rituais de limpeza e higiene $e^{25}$, e sabe-se que lavar as mãos com frequência é um dos comportamentos preventivos preconizados pelas autoridades sanitárias para reduzir as chances de contágio por SARS-CoV-2. Ademais, em muitos pacientes previamente diagnosticados com transtorno obsessivo-compulsivo, pensamentos intrusivos conduzem à prática excessiva do automonitoramento de temperatura corporal para checar a ocorrência de febre - um dos sintomas de COVID-19 - e, em indivíduos sem transtornos mentais pré-existentes, o surgimento de sintomas obsessivo-compulsivos pode ser uma consequência da ansiedade relacionada ao próprio estado de saúde ${ }^{36}$.

O presente estudo revelou associação positiva entre medo relativo à pandemia e sinais e sintomas de comportamento fóbico-evitativo. Como o distanciamento social é uma das medidas mais importantes para evitar a transmissão do SARS-CoV-2, certo nível de comportamento fóbico -evitativo seria esperado no contexto pandêmico vigente. Por outro lado, em crises sanitárias anteriores verificou-se que a predominância de atitudes motivadas diretamente pelo medo frequentemente causa sofrimento psíquico intenso?. Outrossim, comportamento fóbico-evitativo em excesso pode ser um reflexo de uma condição designada como "coronafobia", a qual pode ser definida como um conjunto de reações fisiológicas, cognitivas e comportamentais desencadeadas por preocupações excessivas com um eventual adoecimento por COVID-19 e que causam acentuado prejuízo ao funcionamento em atividades da vida cotidiana ${ }^{37}$.
A exemplo do que se observou no presente estudo, uma pesquisa realizada na Turquia ${ }^{38}$ reportou correlação entre medo relativo à pandemia e sinais e sintomas de estresse $(\mathrm{r}=0,47)$, avaliados, no caso, por meio da Depression, Anxiety and Stress Scale (DASS). Nessa mesma pesquisa, notou-se associação positiva com ansiedade $(r=0,55)$ e depressão $(r=0,38)$. E isso, em linhas gerais, também é compatível com os achados do presente estudo, a julgar pela correlação constatada com ansiedade generalizada. Adicionalmente, é oportuno mencionar que a DASS leva em conta a proximidade conceitual existente entre depressão, ansiedade e estresse, mas contempla cada uma dessas condições como estados emocionais distintos. Sua subescala de estresse, em particular, aborda nervosismo constante, agitação e impaciência $^{39}$, dentre outros sinais e sintomas que podem restringir comportamentos saudáveis ${ }^{40}$, ainda mais em contextos pandêmicos.

Já a associação positiva entre medo e vivência de luto pela pandemia encontrada no presente estudo pode ser explicada considerandose que tal acontecimento funciona como uma comprovação inquestionável de sua letalidade e reforça que a mesma representa um perigo real e, sobretudo, cada vez mais próximo ${ }^{41}$. Parece razoável propor também que o medo da morte por COVID-19 tende a ser intensificado devido às suas especificidades no tocante ao processo de terminalidade. Ocorre que os óbitos geralmente se sucedem em hospitais, com o paciente sedado ou entubado e, para além disso, impossibilitado de receber visitas para evitar o contágio por SARS-CoV-2 entre seus amigos e familiares, o que inviabiliza o empreendimento de rituais presenciais de despedida ${ }^{42}$.

Em suma, conclui-se que esta versão brasileira da FCV-19S mostrou-se adequada quanto às evidências de validade contempladas, de forma que pode ser considerada válida para a avaliação do medo concernente à pandemia atual, bem como potencialmente aproveitável em pandemias futuras devido ao ajuste introduzido nos itens quando da adequação semântica empreendida pelo comitê de especialistas, como já mencionado. Tal instrumento, ademais, é capaz de proporcionar elementos proveitosos para a prática dos profissionais de saúde, pois o medo, vale lembrar, tende a suscitar atitudes disfuncionais quando excessivo ou insuficiente ${ }^{11}$. Logo, o desenvolvimento de intervenções pode se mostrar de grande importância junto a pessoas cujos escores na FCV-19S se situam em um dos dois extremos da distribuição. 
O presente estudo, todavia, possui limitações. Em primeiro lugar, porque os participantes constituíram uma amostra de conveniência de tamanho relativamente reduzido, o que pode ser atribuído ao fato de o recrutamento - justamente devido à pandemia - ter ocorrido apenas de forma online. Em segundo lugar, porque a abordagem qualitativa empregada em prol da obtenção de evidências de validade relativas ao conteúdo pode ter acentuado a natureza subjetiva que já é inerente a esse process ${ }^{31}$. Em terceiro lugar, porque a análise de evidências de validade concernentes à relação com variáveis externas foi operacionalizada, essencialmente, a partir de dados obtidos por meio do QASMP, instrumento que, tendo sido criado para esta oportunidade, não se afigura como "padrão-ouro", embora tenha demonstrado bons indicadores quanto à consistência interna.

Portanto, recomenda-se, em novas pesquisas, que esta versão brasileira da FCV-19S seja aplicada em amostras maiores, e que a obtenção de evidências de validade concernentes ao conteúdo e à relação com outras variáveis envolva, respectivamente, a combinação de procedimentos metodológicos qualitativos e quantitativos e o emprego de instrumentos já consagrados. Além disso, sugere-se que pesquisas futuras se ocupem do estabelecimento de pontos de corte para o instrumento, até porque a publicação em que a FCV-19S foi originalmente apresentada não proporciona tais informações, limitando-se a definir que, quanto mais alto o escore, maior o medo.

\section{Colaboradores}

RS Peres, LT Frick e PA Cortez participaram da concepção, planejamento, coleta, análise e interpretação dos dados, elaboração do texto e aprovação da versão final do artigo. FNFR Queluz, SCS Fernandes, SR Priolo Filho, AC Stelko-Pereira, JZ Martins, JPA Lessa e HMS Veiga participaram da coleta e interpretação dos dados e aprovação da versão final do artigo.

\section{Referências}

1. Neiva MB, Carvalho I, Costa Filho EDS, Barbosa-Junior F, Bernardi FA, Sanches TLM, Oliveira LL, Lima VC, Miyoshi NSB, Alves D. Brazil: the emerging epicenter of COVID-19 pandemic. Rev Soc Bras Med Trop 2020; 21:53:e20200550.

2. Goularte JF, Serafim SD, Colombo R, Hogg B, Caldieraro MA, Rosa AR. COVID-19 and mental health in Brazil: psychiatric symptoms in the general population. J Psychiatr Res 2021; 132:32-37.

3. Domingues CMAS. Challenges for implementation of the COVID-19 vaccination campaign in Brazil. Cad Saude Publica 2021; 37(1):e0034462.

4. Roychowdhury D. 2019 novel coronavirus disease, crisis, and isolation. Front Psychol 2020; 11:1958.

5. Vindegaard N, Benros ME. COVID-19 pandemic and mental health consequences: systematic review of the current evidence. Brain Behav Immun 2020; 89:531-542.

6. Javed B, Sarwer A, Soto EB, Mashwani ZU. The coronavirus (COVID-19) pandemic's impact on mental health. Int J Health Plann Manage 2020; 35(5):993-996.

7. Cortez PA, Joseph SJ, Das N, Bhandari SS, Shoib S Tools to measure the psychological impact of the COVID-19 pandemic: what do we have in the platter? Asian J Psychiatr 2020; 53:102371.

8. Rodríguez-Hidalgo AJ, Pantaleón Y, Dios I, Falla D. Fear of COVID-19, stress, and anxiety in university undergraduate students: a predictive model for depression. Front Psychol 2020; 11:591797.

9. Ornell F, Schuch JB, Sordi AO, Kessler FHP. "Pandemic fear" and COVID-19: mental health burden and strategies. Braz J Psychiatry 2020; 42(3):232-235.

10. Pedrosa AL, Bitencourt L, Fróes ACF, Cazumbá MLB, Campos RGB, de Brito SBCS, Silva ACS. Emotional, behavioral, and psychological impact of the COVID-19 pandemic. Front Psychol 2020; 11:566212. 
11. Mertens G, Gerritsen L, Duijndam S, Salemink E, Engelhard IM. Fear of the coronavirus (COVID-19): predictors in an online study conducted in March 2020. J Anxiety Disord 2020; 74:102258.

12. Harper CA, Satchell LP, Fido D, Latzman RD. Functional fear predicts public health compliance in the COVID-19 pandemic. Int J Ment Health Addict 2020; 1-14.

13. Wise T, Zbozinek TD, Michelini G, Hagan CC, Mobbs D. Changes in risk perception and self-reported protective behaviour during the first week of the COVID-19 pandemic in the United States. $R$ Soc Open Sci 2020; 7(9):200742.

14. Shigemura J, Ursano RJ, Morganstein JC, Kurosawa M, Benedek DM. Public responses to the novel 2019 coronavirus (2019-nCoV) in Japan: mental health consequences and target populations. Psychiatry $\mathrm{Clin} \mathrm{Neu-}$ rosci 2020; 74(4):281-282.

15. Ahorsu DK, Lin CY, Imani V, Saffari M, Griffiths MD, Pakpour AH. The Fear of COVID-19 Scale: development and initial validation. Int $J$ Ment Health Addict 2020; 1-9.

16. Alyami M, Henning M, Krägeloh CU, Alyami H. Psychometric evaluation of the Arabic version of the Fear of COVID-19 Scale. Int J Ment Health Addict 2020; 1-14.

17. Martínez-Lorca M, Martínez-Lorca A, Criado-Álvarez JJ, Armesilla MDC, Latorre JM. The Fear of COVID-19 Scale: validation in Spanish university students. Psychiatry Res 2020; 293:113350.

18. Soraci P, Ferrari A, Abbiati FA, Del Fante E, De Pace R, Urso A, Griffiths MD. Validation and psychometric evaluation of the Italian version of the Fear of COVID-19 Scale. Int J Ment Health Addict 2020: 1-10.

19. Wakashima K, Asai K, Kobayashi D, Koiwa K, Kamoshida S, Sakuraba M. The Japanese version of the Fear of COVID-19 Scale: reliability, validity, and relation to coping behavior. PLoS One 2020; 15(11):e0241958.

20. Pang NTP, Kamu A, Hambali NLB, Mun HC, Kassim MA, Mohamed NH, Ayu F, Rahim SSSA, Omar A, Jeffree MS. Malay version of the Fear of COVID-19 Scale: validity and reliability. Int J Ment Health Addict 2020; $1-10$.

21. Andrade EF, Pereira LJ, Oliveira APL, Orlando DR, Alves DAG, Guilarducci JS, Castelo PM. Perceived fear of COVID-19 infection according to sex, age and occupational risk using the Brazilian version of the Fear of COVID-19 Scale. Death Stud 2020; 1-10.

22. Van de Vijver PJR. Test adaptations. In: Leong FTL, Bartram D, Cheung FM, Geisinger KF, Iliescu D, editores. The ITC international handbook of testing and assessment. Oxford: Oxford University Press; 2016. p. 364-376

23. Beatty PC, Willis GB. Research synthesis: the practice of cognitive interviewing. Public Opin Q 2007; 71(2):287-311.

24. Colina S, Marrone N, Ingram M, Sánchez D. Translation quality assessment in health research: a functionalist alternative to back-translation. Eval Health Prof 2017; 40(3):267-293.

25. American Psychiatric Association (APA). Diagnostic and statistical manual of mental disorders. $5^{\mathrm{a}}$ ed. Washington: American Psychiatric Publishing; 2013.

26. Love J, Selker R, Marsman M, Jamil T, Dropmann D, Verhagen J, Ly A, Gronau QF, Šmíra M, Epskamp S, Matzke D, Wild A, Knight P, Rouder JN, Morey RD, Wagenmakers EJ. Graphical statistical software for common statistical designs. J Stat Softw 2019; 88(2):1-17.
27. Hair JF, Black B, Babin BJ, Anderson RE, Tatham RL. Multivariate data analysis. $6^{a}$ ed. Upper Saddle River: Pearson Prentice Hall; 2006.

28. Forero CG, Maydeu-Olivares A, Gallardo-Pujol D. Factor analysis with ordinal indicators: a Monte Carlo study comparing DWLS and ULS estimation. Struct Equ Model 2009; 16(4):625-641.

29. DunnTJ, Baguley T, Brunsden V. From alpha to omega: a practical solution to the pervasive problem of internal consistency estimation. Br J Psychol 2014; 105(3):399-412.

30. Barrett GV, Phillips JS, Alexander RA. Concurrent and predictive validity designs: a critical reanalysis. J Appl Psychol 1981; 66(1):1-6.

31. Costa ANM, Orpinelli CMZ. Validade de conteúdo nos processos de construção e adaptação de instrumentos de medidas. Cien Saude Colet 2011; 16(7):3061-3068.

32. Hubley AM, Zumbo BD. Validity and the consequences of test interpretation and use. Soc Indic Res 2011; 103(2):219-230.

33. Ågerfalk PJ, Conboy K, Myers MD. Information systems in the age of pandemics: COVID-19 and beyond. Eur J Inform Syst 2020; 29(3):203-207.

34. Centers for Disease Control and Prevention (CDC). Workbook for designing, implementing and evaluating a sharp injury prevention program. Atlanta: CDC; 2008.

35. Snaith RP. The Hospital Anxiety and Depression Scale. Health Qual Life Outcomes 2003; 1:29.

36. Dubey S, Biswas P, Ghosh R, Chatterjee S, Dubey MJ, Chatterjee S, Lahiri D, Lavie CJ. Psychosocial impact of COVID-19. Diabetes Metab Syndr 2020; 14(5):779788.

37. Arora A, Jha AK, Alat P, Das SS. Understanding coronaphobia. Asian J Psychiatr 2020; 54:102384.

38. Satici B, Gocet-Tekin E, Deniz ME, Satici SA. Adaptation of the Fear of COVID-19 Scale: its association with psychological distress and life satisfaction in turkey. Int J Ment Health Addict 2020; 1-10.

39. Henry JD, Crawford JR. The short-form version of the Depression Anxiety Stress Scales (DASS-21): construct validity and normative data in a large nonclinical sample. Br J Clin Psychol 2005; 44(2):227-239.

40. Park CL, Iacocca MO. A stress and coping perspective on health behaviors: theoretical and methodological considerations. Anxiety Stress Coping 2014; 27(2):123137.

41. Goveas JS, Shear MK. Grief and the COVID-19 pandemic in older adults. Am J Geriatr Psychiatry 2020; 28(10):1119-1125.

42. Pattison N. End-of-life decisions and care in the midst of a global coronavirus (COVID-19) pandemic. Intensive Crit Care Nurs 2020; 58:102862.

Artigo apresentado em 22/12/2020

Aprovado em 26/04/2021

Versão final apresentada em 28/04/2021

Editores-chefes: Romeu Gomes, Antônio Augusto Moura da Silva 